\title{
Matching stages of heavy-ion collision models
}

\author{
Yun Cheng, $, 1,2,3,{ }^{*}$ L. P. Csernai, ${ }^{1,2,4}$ V. K. Magas, ${ }^{5}$ B. R. Schlei, ${ }^{6}$ and D. Strottman ${ }^{2,7}$ \\ ${ }^{1}$ Institute of Physics and Technology, University of Bergen, Allegaten 55, N-5007 Bergen, Norway \\ ${ }^{2}$ Frankfurt Institute for Advanced Studies, Johann Wolfgang Goethe University, Ruth-Moufang-Straße 1, \\ D-60438 Frankfurt am Main, Germany \\ ${ }^{3}$ Institute of Particle Physics, Huazhong Normal University, Wuhan 430079, China \\ ${ }^{4}$ MTA-KFKI, Research Inst. of Particle and Nuclear Physics, H-1525 Budapest, Hungary \\ ${ }^{5}$ Departament d'Estructura i Constituents de la Matèria, Universitat de Barcelona, Diagonal 647, E-08028 Barcelona, Spain \\ ${ }^{6}$ GSI Helmholtz Centre for Heavy Ion Research GmbH, Planckstraße 1, D-64291 Darmstadt, Germany \\ ${ }^{7}$ Departamento de Fisica Teorica and IFIC, Centro Mixto Universidad de Valencia CSIC, \\ Institutos de Investigación de Paterna, Aptd. 22085, E-46071 Valencia, Spain
}

(Received 19 April 2010; published 28 June 2010)

\begin{abstract}
Heavy-ion reactions and other collective dynamical processes are frequently described by different theoretical approaches for the different stages of the process, like initial equilibration stage, intermediate locally equilibrated fluid dynamical stage, and final freeze-out stage. For the last stage, the best known is the Cooper-Frye description used to generate the phase space distribution of emitted, noninteracting particles from a fluid dynamical expansion or explosion, assuming a final ideal gas distribution, or (less frequently) an out-of-equilibrium distribution. In this work we do not want to replace the Cooper-Frye description, but rather clarify the ways of using it and how to choose the parameters of the distribution and, eventually, how to choose the form of the phase space distribution used in the Cooper-Frye formula. Moreover, the Cooper-Frye formula is used in connection with the freeze-out problem, while the discussion of transition between different stages of the collision is applicable to other transitions also. More recently, hadronization and molecular dynamics models have been matched to the end of a fluid dynamical stage to describe hadronization and freeze-out. The stages of the model description can be matched to each other on space-time hypersurfaces (just like through the frequently used freeze-out hypersurface). This work presents a generalized description of how to match the stages of the description of a reaction to each other, extending the methodology used at freeze-out, in simple covariant form which is easily applicable in its simplest version for most applications.
\end{abstract}

DOI: 10.1103/PhysRevC.81.064910

PACS number(s): $24 \cdot 10 . \mathrm{Nz}, 25.75 .-\mathrm{q}$

\section{INTRODUCTION}

Relativistic heavy-ion reactions exhibit dominant collective flow behavior, especially at higher energies where the number of involved particles, including quarks and gluons, increases dramatically. At intermediate stages approximate local equilibrium is reached, while the initial and final stages may be far out of local equilibrium. Also, different stages may have different forms or phases of matter, especially when quark-gluon plasma (QGP) is formed.

The need to describe and match different stages of a reaction was realized by the development of the final freeze-out (FO) description in Landau's fluid dynamical (FD) model [1]. Then it was improved by Milekhin [2], and a covariant simple model was given by Cooper and Frye [3]. In all these models the FO happened when the fluid crossed a hypersurface in the space-time.

At early relativistic heavy-ion collisions, the initial compression and thermal excitation was described by a compression shock in nuclear matter. This was already pointed out by the first publications of W. Greiner and E. Teller and their colleagues $[4,5]$, and the shock took place crossing a space-time hypersurface (e.g., a relatively thin layer resulting

\footnotetext{
*yun.cheng@uib.no
}

in a Mach cone). When sudden large changes happen across a space-time front, the conservation laws and the requirement of increasing entropy should be satisfied,

$$
\begin{aligned}
{\left[N^{\mu} d \sigma_{\mu}\right] } & =0 ; \\
{\left[T^{\mu v} d \sigma_{\mu}\right] } & =0 ; \\
{\left[S^{\mu} d \sigma_{\mu}\right] } & \geqslant 0,
\end{aligned}
$$

where $N^{\mu}=n u^{\mu}$ is the baryon current, $S^{\mu}=s u^{\mu}$ is the entropy current, and $T^{\mu \nu}$ is the energy momentum tensor, which, for a perfect fluid, is given by

$$
T^{\mu \nu}=(e+P) u^{\mu} u^{v}-P g^{\mu \nu},
$$

where $e$ is the energy density, $P$ is the pressure, $s$ is the entropy density, and $n$ is the baryon density of matter. These are invariant scalars. The $d \sigma_{\mu}$ is the normal vector of the transition hypersurface, $u^{\mu}$ is the particle four velocity $u^{\mu}=$ $\gamma\left(1, v_{x}, v_{y}, v_{z}\right)=\gamma(1, \vec{v})$, normalized to +1 . The square bracket means $[a]=a_{1}-a_{0}$, the difference of quantity $a$ over the two sides of the hypersurface. The metric tensor is defined as $g^{\mu \nu}=(1,-1,-1,-1)$. We will also use the following notations: $w=e+P, j=N^{\mu} d \sigma_{\mu}$ is the invariant scalar baryon current across the front, $X=(e+P) / n^{2}=w / n^{2}$ is the generalized specific volume, $v^{2}=\vec{v}^{2}=v_{x}^{2}+v_{y}^{2}+v_{z}^{2}$, and $\mu, \nu \cdots=0,1,2,3, i, j \cdots=1,2,3=x, y, z$. 
For a perfect fluid, local equilibrium is assumed, thus the fluid can be characterized by an equation of state (EoS), $P=$ $P(e, n)$. Equations (1) and (2) and the EoS are six equations and can determine the six parameters of the final state, $e, n$, $P$, and $\vec{v}$.

Later Csernai [6,7] pointed out the importance of satisfying energy, momentum, and particle-charge-conservation laws across such hypersurfaces and generalized the earlier description of Taub [8] to spacelike and timelike hypersurfaces (with spacelike and timelike normals, respectively). In this situation the matter both before and after the shock was near thermal equilibrium, and thus the conservation laws led to scalar equations connecting thermodynamical parameters of the two stages of the matter: the generalized Rayleigh line and Taub adiabat $[6,7]$,

$$
j^{2}=[P]\left(d \sigma^{\mu} d \sigma_{\mu}\right) /[X], \quad[P]=[(e+P) X] /\left(X_{1}+X_{0}\right) .
$$

At much higher energies, at the first stages of the collision, the matter becomes "transparent" and the initial state is very far from thermal equilibrium. For this stage other models were needed to handle the initial development (e.g., Ref. [9]). The initial nonequilibrium state in this situation cannot be characterized by thermodynamical parameters or an EoS, so the previous approach, with the generalized Rayleigh line and Taub adiabat is not applicable. Nevertheless, the intermediate (FD) stage is in equilibrium and has an EoS, while the initial state has a well-defined energy momentum tensor. In this work we demonstrate that the final invariant scalar, thermodynamical parameters can be determined in this situation also from the conservation laws.

Then, Bugaev [10,11] observed that FO across hypersurfaces with spacelike normals, has problems with negative contributions in the Cooper-Frye evaluation [3] of particle spectra; thus, the FO must yield an anisotropic distribution, which he could approximate with a cut-Jüttner distribution $[10,11]$. This is not surprising as in the rest frame of the front (RFF) all post-FO particles must move "outward"; that is, $p^{\mu} d \sigma_{\mu}>0$ is required. This condition is not satisfied by any noninteracting thermal equilibrium distribution, which extend to infinity in all directions, even if they are boosted in the RFF. ${ }^{1}$

Subsequently, another analytic form was proposed by Csernai and Tamosiunas, the canceling-Jüttner distribution [12], which replaced the sharp cutoff by a continuous cutoff, based on kinetic model results.

Parallel to this development, the FO process was analyzed in kinetic, transport approaches [13-17], where the FO happened in an outer layer of the space-time, or in principle it could be extended to the whole fluid (although, at early moments of a collision or explosion, from the center of the reaction few particles can escape). These transport studies also indicated that the post-FO distributions may become anisotropic [14-16] even for FO hypersurfaces with timelike normal (in short,

\footnotetext{
${ }^{1}$ In the following discussion we use the term anisotropic distribution for momentum distributions in their own local rest (LR) frame. Thermal distributions are spherical in their LR frame, although they become anisotropic in another frame of reference [7].
}

timelike surface) if the normal, $d \hat{\sigma}^{\mu}$, and the velocity four vector, $u^{\mu}$, are (very) different.

These studies led to another FO description, where the initial stages of the collision with strongly interacting matter were described by fluid dynamics, while the final, outer space-time domain (or later times) was described by weakly interacting particle (and string) transport models, where the final FO was inherently included, as each particle was tracked, until its last interaction. It is important to mention that in these approaches the transition from the FD stage to the molecular dynamics (MD) or cascade stage happens when the matter crosses a space-time hypersurface; thus, the conservations laws [6,7] have to be satisfied and the post-FO particle phase space distributions $[10,12]$ have to be used when the post-FO distributions become anisotropic.

In this work, for the first time we present a simple covariant solution for the transition problem and conservation laws for the situations when the matter after the front is in thermal equilibrium (i.e., it has isotropic phase space distribution) and has an EoS, but the matter before the front must not be in an equilibrium state.

Then we discuss the situation where microscopic models are appended to the FD model, which are in or close to thermal equilibrium, but the EoS is not necessarily known.

Subsequently, we present the way to generalize the problem to anisotropic matter in final state, which is necessary for FO across spacelike surfaces and also for timelike surfaces if the flow velocity is large in the RFF. This problem was solved in kinetic approach for the Bugaev cut-Jüttner approach $[11,13]$ and the Csernai-Tamosiunas canceling-Jüttner approach [12], by calculating the energy momentum tensors explicitly from the anisotropic phase space distributions, but no general solution is given for post-FO matter with anisotropic pressure tensor.

\section{NUMERICAL EXTRACTION OF A FREEZE-OUT HYPERSURFACE}

The transition hypersurface between two stages of a dynamical development are most frequently postulated, governed by the requirement of simplicity. Thus, such a hypersurface is frequently chosen as a fixed coordinate time in a Descartian frame $t$ or at a fixed proper time $\tau$ from a space-time point, although in a general $(3+1)$-dimensional system the choice of such a point is not uniquely defined. It is important that the transition hypersurface should be continuous (without holes where conserved particles or energy or momentum could escape through without being accounted for). To secure that one quantity (e.g., baryon charge) does not escape through the holes of a hypersurface is not sufficient, because other quantities may (e.g., momentum in case if $P d V$ is different on the two sides of a hole). Again, to construct such a continuous hypersurface in a general $(3+1)$-dimensional system is a rather complex task, although, in $1+1$ or $2+1$ dimensions it seems to be easy.

Both the initial-state models and the intermediate-stage, FD models may be such that the calculation could be continued beyond the point where a transition takes place. Then spacetime location of the transition to the next stage can or should be 

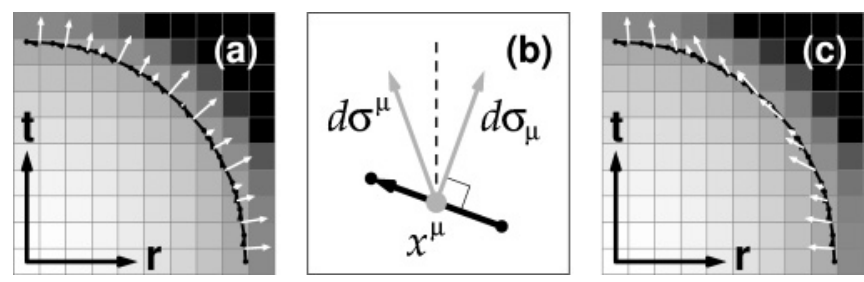

FIG. 1. (a) A gray-level image representing the temperature evolution in space-time of a 1D relativistic fluid (see text) which is superimposed with contravariant FO contour vectors (black) and with corresponding covariant normal vectors $d \sigma_{\mu}\left(x^{\mu}\right)$ (white). (b) Covariant and contravariant normal vectors, $d \sigma_{\mu}\left(x^{\mu}\right)$ and $d \sigma^{\mu}\left(x^{\mu}\right)$ (gray), respectively, which originate at the contravariant center $x^{\mu}$ (gray) of a single contravariant FO contour vector (black). (c) As in (a), but with contravariant normal vectors $d \sigma^{\mu}\left(x^{\mu}\right)$ (white) instead of covariant ones.

decided, based on a physical condition or requirement, which may be external to the development itself. As a consequence, in some cases the determination of transition surface may be an iterative process.

Numerically, the extraction of a FO hypersurface is by no means trivial. One of us (B.R.S.) has recently provided a proper numerical treatment regarding the extraction of FO hypersurfaces in two (2D), three (3D), and four (4D) dimensions [18-21].

For instance, in $2 \mathrm{D}$ the history, that is, the temporal evolution, of a temperature field of a one-dimensional (1D) relativistic fluid can be represented by a gray-level image (cf. Fig. 1). In the figure, we use the time $t$ and the radius $r$ for the temporal and the spatial dimensions, respectively. Let bright pixels (i.e., picture elements) refer to high temperatures and dark ones to low temperatures of the fluid. In this example, a 2D FO hypersurface is an isotherm.

In Fig. 1(a), we also depict the corresponding covariant normal vectors $d \sigma_{\mu}\left(x^{\mu}\right)$. In 2D, the length of each normal vector is equal to the length of each supporting isocontour vector. Each normal vector has its origin at the contravariant center, $x^{\mu}$, of a given contravariant isocontour vector and points to the exterior of the enclosed space-time region. The latter is also indicated in Fig. 1(b), where we show that a contravariant normal vector $d \sigma^{\mu}\left(x^{\mu}\right)$ can be obtained by reflection of the covariant normal vector $d \sigma_{\mu}\left(x^{\mu}\right)$ at the time axis (dashed line).

Finally, in Fig. 1(c) we show the contravariant FO contour vectors with their corresponding contravariant normal vectors $d \sigma^{\mu}\left(x^{\mu}\right)$. Not all of these contravariant normal vectors point to the exterior of the enclosed space-time region.

Note that the sign conventions of the normals of the transition hypersurface are important and must be discussed, especially if both timelike and spacelike surfaces are studied. In fact, only the timelike contravariant normal vectors point outward, whereas the spacelike contravariant normal vectors point inward. ${ }^{2}$

\footnotetext{
${ }^{2}$ Note that we actually use for hypersurface construction in $(1+$ $1)$-, $(2+1)$-, and $(3+1)$-dimensional numerical simulations the corresponding computer codes, that is, DICONEX, VESTA, and STEVE,
}

If we know the FO hypersurface and the local momentum distribution after the transition the total, measurable momentum distribution can be evaluated by the Cooper-Frye formula [3].

\section{EQUATIONS FOR PARAMETERS OF FINAL MATTER IN EQUILIBRIUM}

Let us define the contravariant and covariant surface normal four vectors as

$$
\begin{aligned}
d \sigma^{\mu} & =\left(\sigma_{t}, \sigma_{x}, \sigma_{y}, \sigma_{z}\right)=\left(\sigma_{t}, \vec{\sigma}\right), \\
d \sigma_{\mu} & =\left(\sigma_{t},-\sigma_{x},-\sigma_{y},-\sigma_{z}\right)=\left(\sigma_{t},-\vec{\sigma}\right),
\end{aligned}
$$

where in general $d \sigma^{\mu} d \sigma_{\mu}= \pm D^{2}$, as the surface element can be either spacelike $(-)$ or timelike $(+)$. We can also introduce a unit normal to the surface as $d \hat{\sigma}^{\mu} \equiv d \sigma^{\mu} / D$ so that $d \hat{\sigma}^{\mu} d \hat{\sigma}_{\mu}= \pm 1$. Furthermore, $d \hat{\sigma}^{\mu}=\gamma_{\sigma}\left(1, s_{x}, s_{y}, s_{z}\right)=$ $\gamma_{\sigma}(1, \vec{s})$, where for timelike surfaces $\gamma_{\sigma}^{2}=1 /\left(1-\vec{s}^{2}\right)$ and for spacelike surfaces $\gamma_{\sigma}^{2}=1 /\left(\vec{s}^{2}-1\right)$. For the frequently used timelike 1D case $d \hat{\sigma}^{\mu}=\gamma_{\sigma}(1, \vec{s})=\gamma_{\sigma}(1,0,0, d t / d z)$.

In the general case, the conserved energy-momentum current crossing the surface element is

$$
A^{\mu}=T^{\mu \nu} d \sigma_{v}=w u^{\mu} u^{v} d \sigma_{v}-P g^{\mu v} d \sigma_{\nu} .
$$

$A^{\mu}$ must be continuous across the FO surface, as must the baryon current $N^{\mu} d \sigma_{\mu}$,

$$
j=N^{\mu} d \sigma_{\mu}=n u^{\mu} d \sigma_{\mu}=n \gamma\left[\sigma_{t}-(\vec{v} \cdot \vec{\sigma})\right],
$$

where $j$ is the invariant scalar baryon charge current.

We assume that the initial state, " 0 ," and its energy momentum tensor and baryon current before the front is known. We aim for the characteristics of the final state. In total, there are six unknowns in the equilibrated final state; these are three-vector $\vec{v}, e, P$, and $n$ (here we drop the index "1" for the final state for shorter notation); however, the pressure $P$, a function of $e$ and $n$, is given by the $\operatorname{EoS}, P=P(e, n)$. Knowing $n$ and $e$, the EoS and the particular form of the corresponding equilibrated distribution function, the parameters $T$ and $\mu$, can also be obtained.

Thus, we have to solve five equations:

$$
\begin{aligned}
j_{0} & =n \gamma\left[\sigma_{t}-(\vec{v} \cdot \vec{\sigma})\right], \\
A_{0 t} & =w \gamma^{2}\left[\sigma_{t}-(\vec{v} \cdot \vec{\sigma})\right]-P \sigma_{t}, \\
A_{0 x} & =w \gamma^{2}\left[\sigma_{t}-(\vec{v} \cdot \vec{\sigma})\right] v_{x}-P \sigma_{x},
\end{aligned}
$$

respectively. Reference [18] explains in great detail the extraction of an oriented FO contour, which is represented by a set of contravariant (so-called "DICONEX isocontour") vectors. In 2D, the simplices or simplexes which represent a hypersurface best are line segments, whereas in 3D and 4D they are triangles [19,21] and tetrahedrons [20], respectively. In particular, the contravariant 2D FO contour vectors are oriented counterclockwise around the enclosed space-time regions. The covariant normals of the contravariant simplices are obtained from calculating the mathematical duals of these simplices with respect to a geometric product (cf., e.g., Ref. [22]) within the $N$-dimensional multilinear space under consideration. Note that the covariant normal vectors do not depend on any given metric tensor, whereas the contravariant normal vectors do [21]. 


$$
\begin{aligned}
& A_{0 y}=w \gamma^{2}\left[\sigma_{t}-(\vec{v} \cdot \vec{\sigma})\right] v_{y}-P \sigma_{y}, \\
& A_{0 z}=w \gamma^{2}\left[\sigma_{t}-(\vec{v} \cdot \vec{\sigma})\right] v_{z}-P \sigma_{z} .
\end{aligned}
$$

The left-hand sides represent quantities of the initial state of matter and the corresponding conserved quantities are known. Equations (9) and (10) can be solved for $\gamma^{2}$ in the calculational frame

$$
\gamma^{2}=\frac{A_{0 t}+P \sigma_{t}}{w\left[\sigma_{t}-(\vec{v} \cdot \vec{\sigma})\right]}, \quad \gamma^{2}=\frac{A_{0 x}+P \sigma_{x}}{w v_{x}\left[\sigma_{t}-(\vec{v} \cdot \vec{\sigma})\right]} .
$$

Using now Eqs. (10)-(12), one obtains $v_{x}$ and in a similar fashion $v_{y}$ and $v_{z}$ :

$$
v_{x}=\frac{A_{0 x}+P \sigma_{x}}{A_{0 t}+P \sigma_{t}}, \quad v_{y}=\frac{A_{0 y}+P \sigma_{y}}{A_{0 t}+P \sigma_{t}}, \quad v_{z}=\frac{A_{0 z}+P \sigma_{z}}{A_{0 t}+P \sigma_{t}} .
$$

This results for $\gamma^{2}=1 /\left(1-\vec{v}^{2}\right)$, in

$$
\gamma^{2}=\frac{\left(A_{0 t}+P \sigma_{t}\right)^{2}}{\left(A_{0}^{\mu}+P d \sigma^{\mu}\right)^{2}},
$$

where $\quad\left(A_{0}^{\mu}+P d \sigma^{\mu}\right)^{2}=\left(A_{0 t}+P \sigma_{t}\right)^{2}-\left(A_{0 x}+P \sigma_{x}\right)^{2}-$ $\left(A_{0 y}+P \sigma_{y}\right)^{2}-\left(A_{0 z}+P \sigma_{z}\right)^{2}$ is an invariant scalar, and $\gamma$ transforms as the 0th component of the four-vector $A_{0}^{\mu}+P d \sigma^{\mu}$. Notice that Eq. (8) was not used up to this point; thus, we can use there results both for the baryon-free and the baryon-rich cases.

We can have an elegant direct solution for the proper energy density, $e$, and pressure, $P$, as both of these quantities are invariant scalars, and we can express these by the covariant, four-vector Eq. (6). From this four-vector equation we can get two invariant scalar equations by (i) taking its norm, $A_{0}^{\mu} A_{0 \mu}$, and (ii) taking its projection to the normal direction, $A_{0}^{\mu} d \sigma_{\mu}$ :

$$
\begin{aligned}
A_{0}^{\mu} A_{0 \mu}= & w^{2}\left(u^{\mu} d \sigma_{\mu}\right)^{2}+P^{2}\left(d \sigma^{\mu} d \sigma_{\mu}\right) \\
& -2 P w\left(u^{\mu} d \sigma_{\mu}\right) u_{\mu} g^{\mu v} d \sigma_{\nu} \\
= & w(e-P)\left(u^{\mu} d \sigma_{\mu}\right)^{2}+P^{2}\left(d \sigma^{\mu} d \sigma_{\mu}\right), \\
A_{0}^{\mu} d \sigma_{\mu}= & w\left(u^{\mu} d \sigma_{\mu}\right)^{2}-P\left(d \sigma^{\mu} d \sigma_{\mu}\right) .
\end{aligned}
$$

Now expressing $w\left(u^{\mu} d \sigma_{\mu}\right)^{2}$ from Eq. (17) and inserting it to Eq. (16), we obtain our final equation,

$$
A_{0}^{\mu} A_{0 \mu}=(e-P) A_{0}^{\mu} d \sigma_{\mu}+e P\left(d \sigma^{\mu} d \sigma_{\mu}\right),
$$

which can be solved straightforwardly if the EoS, $P=P(n, e)$, is known. The other three elements of the equation, $A_{0}^{\mu} A_{0 \mu}$, $A_{0}^{\mu} d \sigma_{\mu}$, and $d \sigma^{\mu} d \sigma_{\mu}$, are known from the normal to the surface and from energy-momentum current from the pretransition side.

Then, Eqs. (13)-(15) can be used to determine the final flow velocity. At the end, after all conservation law equations are solved, we have to check the nondecreasing entropy condition (3) to see whether the solution is physically possible. If the overall entropy is decreasing after transition, that would mean that the hypersurface is chosen incorrectly. One will need to choose more realistic condition for the transition and repeat the calculations.

This result can be used both if the initial state is in equilibrium and if it is not.

\section{A. Final matter with zero Baryon charge}

In case of an ideal gas of massless particles after the front, with an $\operatorname{EoS}$ of $P=e / 3$, Eq. (18) leads to a quadratic equation

$$
d \hat{\sigma}^{\mu} d \hat{\sigma}_{\mu} e^{2}+2 a^{\mu} d \hat{\sigma}_{\mu} e-3 a^{\mu} a_{\mu}=0,
$$

where $a^{\mu} \equiv A_{0}^{\mu} / D$ is the energy momentum transfer four vector across a unit hypersurface element.

If the flow velocity is normal to the FO hypersurface, $u^{\mu}=d \hat{\sigma}^{\mu}$, then for an initial perfect fluid in the LR frame the preceding covariant equation takes a simple form,

$$
e^{2}+2 e_{0} e-3 e_{0}^{2}=0 .
$$

This has two real roots, $e=e_{0}$ (energy density is conserved) and $e=-3 e_{0}$, which does not correspond to a physical solution, as the energy density should not be negative.

\section{B. Final matter with finite Baryon charge}

If the EoS depends on the conserved baryon charge density also, then we must exploit in addition Eq. (7)

$$
j_{0} \equiv j=n\left(u^{\mu} d \sigma_{\mu}\right),
$$

and inserting $u^{\mu} d \sigma_{\mu}=j / n$ from here to Eq. (17) yields

$$
j^{2} \frac{w}{n^{2}}=A_{0}^{\mu} d \sigma_{\mu}+P\left(d \sigma^{\mu} d \sigma_{\mu}\right)
$$

where $w / n^{2}=X$ is the generalized specific volume, well known from relativistic shock and detonation theory [7]. This equation provides another equation for $e+P$ as

$$
\frac{e+P}{n^{2}}=\frac{1}{j^{2}}\left[A_{0}^{\mu} d \sigma_{\mu}+P\left(d \sigma^{\mu} d \sigma_{\mu}\right)\right]
$$

which, together with Eq. (18) and the EoS, $P=P(e, n)$, provide three equations to be solved for $e, P$, and $n$.

This evaluation of the post-FO configuration is in agreement with the theory of relativistic shocks and detonations $[6,8]$, allowing for both spacelike and timelike FO hypersurfaces (see also [7]). This method of evaluation observables is frequently used at the end of FD model calculations (see, e.g., $[23-25])$.

\section{TRANSITION TO MOLECULAR DYNAMICS BEFORE FREEZE-OUT}

Recently, a frequently practiced method of describing the final stages of a reaction is to switch the FD model over to a MD description at a transition hypersurface. This is frequently a fixed time, $t$, or fixed proper time, $\tau$, hypersurface. The generation of the initial state of such an MD model is a task that depends on the constituents of the matter described by the MD model. Nevertheless, same principles must be satisfied, like the conservation laws [Eqs. (1) and (2)].

\section{A. Equilibrium and EoS known before and after the transition}

Let us assume, although not required by physical laws, that we have thermal equilibrium on both sides of the transition 
and we know explicitly the corresponding final momentum distribution of particles. Then, the fundamental equation for constructing the post-transition microscopic state, in addition to the conservation laws, is the Cooper-Frye formula,

$$
E \frac{d N_{i}}{d^{3} p}=\int_{\sigma} f_{i}(x, p) p^{\mu} d \sigma_{\mu},
$$

assuming that the local phase space distribution, $f_{i}(x, p)$, is known for all initial components of the MD model. If $f_{i}(x, p)$ are local equilibrium distributions, then (in principle) we know the intensive and extensive thermodynamical parameters and the EoS of the matter when the MD model simulation starts. These must not be the same as the ones before the transition hypersurface.

In the usual transition from FD to MD models, where the initial state of MD is in equilibrium, the EoSs are known on both sides of the transition hypersurface, ${ }^{3}$ and thus, both the equations of Rayleigh-line and Taub-adiabat [Eqs. (5)], as well as the invariant scalar equations derived here, Eqs. (16)-(19) can be used to determine all parameters of the matter starting the MD simulation. These then determine the phase space distributions, $f_{i}(x, p)$ of all components of the MD simulation. Subsequently, Eq. (20) can be used to generate randomly the initial constituents of the MD simulation.

As Eq. (20) is a covariant equation applicable in any frame of reference, the most straightforward is to perform the generation of particles in the calculational frame of the MD model. This transition is by now performed in many hybrid models combining fluid dynamics with microscopic transport models [26]. These models at present are the most effective for describing experimental data and make the need for a modified Boltzmann transport equation [16] less problematic.

In some cases, the first step of the transition, the determination of the parameters of the final state from the exact conservation laws, is dropped with the argument that both before and after the transition the matter has the same constituents and the same EoS; thus, the all extensive and intensive thermodynamical parameters as well as the flow velocity must remain the same. Then, using the intensive parameters, the final particle distributions in the Cooper-Frye formula [Eq. (20)] can be directly evaluated in a straightforward way. This procedure is correct, but only if all features of the two states of the matter and their EoSs are identical. In some cases the pretransition EoS assumes effective hadron masses depending on the matter density, while the final $\mathrm{EoS}$ is that of a hadron ideal gas mixture, but with fixed vacuum masses. This leads to a difference in the EoS; thus, the preceding procedure is approximate. In such cases, the method can be used, but the accurate conservation laws can be enforced by a final adjustment step described in the next section.

The situation is similar if the constituents and the EoSs are almost identical before and after the transition, but before

\footnotetext{
${ }^{3}$ One should pay attention that the choice of the hypersurface should be continuous and should not have holes. In $3+1 \mathrm{D}$ this is a nontrivial problem, which is solved by the methods DICONEX, VESTA and STEVE, mentioned previously.
}

the transition a weak or weakening mean-field potential or compression energy is taken into account.

\section{B. Enforcing conservation laws with approximate generation of the final state}

In addition to the aforementioned approximate methods, even for really identical EoSs across the transition or with generating the final EoS parameter based on conservation laws for the final EoS, inaccuracies may arise owing to other reasons: During the random generation of the initial constituent particles of the MD simulation, the exact conservation laws may be violated, owing to finite-number effects. However, the energy and particle-number conservations are usually enforced during the random generation of particles, even if the preceding procedure for solving the conservation laws beforehand is not fully followed. This is usually the consequence of the fact that the EoS of the MD model is not necessarily known if the model has complex constituents and laws of motion.

In any case, to remedy this random error and make the conservation laws exactly satisfied, a final correction step is advisable, and it is not always performed. If the energy and particle-number conservations are enforced then, the last variable to balance is the momentum conservation. This regulates the flow velocity of the matter after the transition initiating the MD simulation.

The energy momentum tensor and baryon current for the generated random set of particle species, $i$, for each fluid cell (or group of cells if the multiplicity in a single cell is too low) can be calculated from the kinetic definition

$$
\begin{aligned}
T^{\mu \nu}(x) & =\sum_{i} \int \frac{d^{3} p_{i}}{p_{i}^{0}} p_{i}^{\mu} p_{i}^{v} f_{i}\left(x, p_{i}\right), \\
N^{\mu}(x) & =\sum_{i} \int \frac{d^{3} p_{i}}{p_{i}^{0}} p_{i}^{\mu} f_{i}\left(x, p_{i}\right),
\end{aligned}
$$

which yield the resulting momentum and flow velocity of the matter. This can be used to adjust the flow velocity to achieve exact conservation of momentum and modify the velocity of generated particles by the required Lorentz boost. The other conserved quantities may then be affected also, but an iterative procedure for eliminating the error completely is not crucial because the error can be given quantitatively.

If the randomly generated state is not following a thermal equilibrium phase space distribution, $f_{i}\left(x, p_{i}\right)$, and thus does not have an EoS, the previously described scalar equations cannot be used to generate the initial configuration of the MD model. Nevertheless, the second step to checking the conservation laws with the kinetic definition and then correcting the parameters of the generated particles can be done. For a required level of accuracy in this case an iterative procedure may be necessary.

Another, easier way to remedy this problem is to choose the transition hypersurface earlier so that the subsequent matter is still in thermal equilibrium. This can always be done if the requirement of entropy increase is satisfied. 

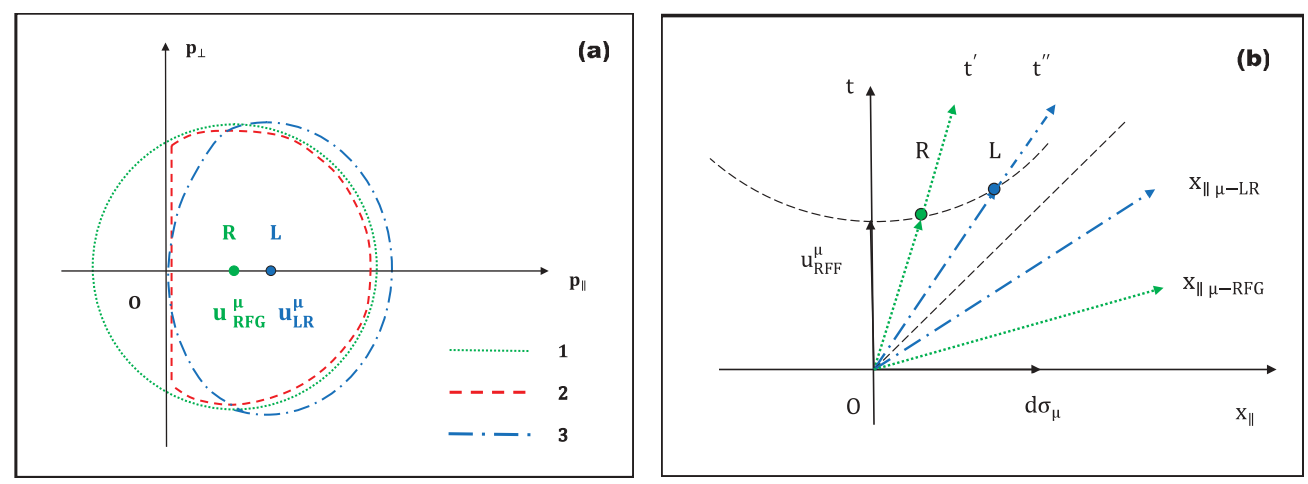

FIG. 2. (Color online) (a) Illustrative contour plot of momentum distributions of particles after FO to an anisotropic final state. Here the $p_{\|}$axis points in the direction of the spacelike normal of the FO hypersurface, while $p_{\perp}$ is the orthogonal direction to $p_{\|}$. Line 1 indicates the uncut Jüttner distribution (dotted-line), line 2 indicates the cut-Jüttner distribution (dashed-line), and line 3 indicates the canceling-Jüttner distribution (dashed-dotted line). $\mathrm{R}$ is the center of the spherical uncut Jüttner distribution moving with velocity, $u_{\mathrm{RFG}}^{\mu}$, and $\mathrm{L}$ is the center of the cut- and canceling-Jüttner distributions, which move to the right along the parallel momentum direction with velocity, $u_{\mathrm{LR}}^{\mu}$, corresponding to their LR frame. If $u_{\mathrm{RFG}}^{\mu}$ and $u_{\mathrm{LR}}^{\mu}$ have a nonvanishing $\perp$ component, the resulting distribution can be obtained by an additional Lorentz boost in that direction. (b) Space-time reference frames for anisotropic final state distributions. The time axis, $t$, and the spatial axis, $x_{\|}$, represent the rest frame of front (RFF), which is the defining front for the cut-Jüttner and canceling-Jüttner distributions. L indicates the local rest frame (LR) of the post-FO cut- or canceling-Jüttner distribution. R indicates the rest frame of gas (RFG) corresponding to the isotropic, uncut Jüttner distribution. Thus, the velocities $u_{\mathrm{RFF}}^{\mu}, u_{\mathrm{RFG}}^{\mu}$, and $u_{\mathrm{LR}}^{\mu}$ point in the directions of the time axes of the corresponding reference frames, indicated by $t, t^{\prime}, t^{\prime \prime}$, respectively. $d \sigma_{\mu}$ is the normal vector of the front; it has the same direction as the spatial axis, $x_{\|}$. The spatial axes of the frames RFG (R) and LR (L) are shown in the same line style (color) as the corresponding distributions. The LR frames for cases 2 and 3 are the same.

\section{FINAL STATE OUT OF THERMAL EQUILIBRIUM}

We have mentioned that the assumption for having thermal equilibrium in the final state is neither excluded nor required from transport theoretical considerations. However, thermal equilibrium distribution is not possible if we have to describe FO across a spacelike hypersurface (see the discussion in Sec. I).

In the MD model description the final post-FO momentum distributions develop a local anisotropy if the FO has locally a preferred direction. Unless the unit normal of the $\mathrm{FO}$ hypersurface is equal to the local flow velocity of the pre-FO matter, there is always a selected spatial direction which is the dominant direction of FO. This situation is discussed in several theoretical works, and some general features can be extracted from these studies.

\section{A. Approximate kinetic models for freeze-out}

In explicit transport models this situation is handled [10-13]: Starting from an equilibrium Jüttner distribution and considering a momentum dependent escape probability in the collision term-which reflected the direction of the FO front and the distance from the front-an anisotropic distribution was obtained (i.e., a distribution, which was anisotropic even in its own LR frame).

This anisotropic distribution could be approximated with analytic distribution functions [10,11]: The starting point is the uncut, isotropic, Jütner distribution in the rest frame of the gas (RFG), which is centered around the four-velocity vector, $u_{\mathrm{RFG}}^{\mu}$. This distribution is then cut or cut and smoothed. The resulting distribution has a different new flow velocity,
$u_{L R}^{\mu}$, which is nonzero in RFG, and is pointing in space in the direction of the normal of the FO hypersurface, $\vec{\sigma}$, labeled with $\|$. This $u_{\mathrm{LR}}^{\mu}$ defines the LR frame of the post FO matter.

The spatial direction of $\vec{\sigma}$ is not affected by the Lorentz transformation from RFF to RFG and then to LR, as $\vec{\sigma}$ is the direction of the Lorentz transformation from RFG to LR. ${ }^{4}$ In the general case the boost in the $\vec{v}_{\mathrm{RFG}, \perp}$ direction leads to a change of the distribution function in the $\vec{p}_{\perp}$ direction, but does not affect the distribution in the $\vec{p}_{\|}$direction or the procedure of cutting or canceling the distribution in the $\|$ direction. [The illustration in Fig. 2(a) shows the spatial momentum distribution where the boost in the orthogonal direction $\vec{v}_{\mathrm{RFG}, \perp}$ is already performed.

In the final LR frame, the matter is characterized by a rather complex energy momentum tensor, inheriting some parameters from the original uncut distribution in RFG, like the temperature and chemical potential, but as the resulting distribution is not a thermal equilibrium distribution, these parameters are not playing any thermodynamical role. One has to determine all parameters numerically from conservation laws [Eqs. (1) and (2)], as done in Refs. [11,12].

Interestingly, a simplified numerical kinetic FO model [13] led to a FO distribution satisfying the condition $p^{\mu} d \sigma_{\mu}>0$ for spacelike FO with a smooth distribution function, which is anisotropic (also in its own LR frame) and has a symmetry axis pointing in the dominant FO direction. This distribution

\footnotetext{
${ }^{4}$ In general, the spatial components of the FO surface, $\vec{\sigma}$, must not be parallel to $\vec{v}_{\mathrm{RFG}}$ or $\vec{v}_{\mathrm{LR}}$, but these latter velocities can be decomposed to $\|$ and $\perp$ components with respect to $\vec{\sigma}$. Owing to the construction [10-12] of the cut- or canceling-Jüttner distributions $\left(\vec{v}_{\mathrm{LR}}-\vec{v}_{\mathrm{RFG}}\right) \|$ $\vec{\sigma}$ or $\vec{v}_{\mathrm{LR}, \perp}=\vec{v}_{\mathrm{RFG}, \perp}$.
} 
was then approximated with an analytic, "cancelling-Jüttner" distribution [12], which can also be used to solve the FO problem.

After FO, the symmetry properties of the energy momentum tensor are the same for the cut-Jüttner and canceling-Jüttner cases [10-12]. The FO leads to an anisotropic momentum distribution and therefore to an anisotropic pressure tensor. The energy momentum tensor is not diagonal in the RFG frame; there is a nonvanishing transport term, $T^{0 i}[11,12]$, in the 2D plane spanned by the four vectors, $u_{\mathrm{RFG}}^{\mu}$ and $d \hat{\sigma}_{\mu}$. One can, however, diagonalize the energy momentum tensor by making a Lorentz boost into the LR frame using Landau's definition for the four velocity, $u_{\mathrm{LR}}^{\mu}$. In this frame then the energy momentum tensor becomes diagonal, but the pressure terms are not identical, owing to the anisotropy of the distribution:

$$
T^{\mu \nu}=\left.\operatorname{diag}\left(e, P_{\|}, P_{\perp}, P_{\perp}\right)\right|_{\mathrm{LR}} .
$$

Here the energy density, $e$, of course must not be the same as in the case of an isotropic, thermal equilibrium post-FO momentum distribution. This can be seen from the kinetic definition of the energy momentum tensor, as shown in Refs. [11,12].

We need the complete post-FO momentum distribution and the corresponding energy momentum tensor to determine final observables. This depends on the transport processes at FO and cannot be given in general; however, owing to the symmetries of the collision integral, the symmetries of the energy momentum tensor are the same irrespective of the ansatz used (e.g., cut-Jüttner, canceling-Jüttner, or some other distribution).

In kinetic transport approaches, the microscopic escape probability [15] is peaking in the direction of $d \hat{\sigma}_{\mu}$, which yields a distribution peaking in this direction, that is, yielding the same symmetry properties as the previously mentioned analytic ansatzes. The energy momentum tensor in general takes the form

$$
T^{\mu \nu}=e u_{\mathrm{LR}}^{\mu} u_{\mathrm{LR}}^{v}-P_{\perp} \Delta_{\mathrm{LR}}^{\mu \nu}+\left(P_{\|}-P_{\perp}\right) \hat{F}^{\mu} \hat{F}^{v},
$$

where $\Delta_{\mathrm{LR}}^{\mu \nu}$ is the orthogonal projector to $u_{\mathrm{LR}}^{\mu}$ and $\hat{F}_{\mu}$ is the unit four-vector projection of $d \sigma_{\mu}$ in the direction orthogonal to $u_{\mathrm{LR}}^{\mu}$; that is, $\hat{F}^{\mu}=C \Delta_{\mathrm{LR}}^{\mu \nu} d \sigma_{v}$, where $C$ ensures normalization to -1 . In the Landau LR frame, this returns expression (21). The four velocity, $u_{\mathrm{LR}}^{\mu}$, and the other parameters of the post-FO state of matter should be determined from the conservation laws [Eqs. (1) and (2)]. The schematic diagram of the asymmetric distributions and the different reference frames can be seen in Fig. 2.

The FO problem was solved for these configurations and ansatzes by satisfying the conservation laws explicitly for the full energy momentum tensor. We do not have a general $\operatorname{EoS}(\mathrm{s})$ that would characterize the connection among $e, P_{\|}$, and $P_{\perp}$; furthermore, the relation connecting these quantities depends on the four vectors $d \hat{\sigma}^{\mu}$ and $u_{\mathrm{LR}}^{\mu}$. In addition, this connection depends on the details or assumptions of the transport model. The simple models [11,12] provide examples for such a dependence. If $d \hat{\sigma}^{\mu}$ is known, then for baryon-free matter we can determine four unknowns: $u_{\mathrm{LR}}^{\mu}$ and an additional parameter of the post-FO distribution from Eq. (2). (Owing to normalization, only three components of $u_{\mathrm{LR}}^{\mu}$ are unknowns.)
For baryon-rich matter we can determine one more unknown parameter, because we have one additional equation, the conservation of baryon charge from Eq. (1).

\section{B. Exploiting general symmetries of anisotropic final states}

The first step of solution can be done similarly to the isotropic case. Then in Eq. (6) the enthalpy will change as $w \rightarrow e+P_{\perp} \equiv w_{\perp}$ and $P \rightarrow P_{\perp}$; plus an additive term will appear, $\left(P_{\|}-P_{\perp}\right) \hat{F}^{\mu} \hat{F}^{v} d \sigma_{\nu}$. Furthermore, Eqs. (9)-(12) remain of the same form, with $w_{\perp}$ and $P_{\perp}$; plus the additive term $\left(P_{\|}-P_{\perp}\right) \hat{F}^{\mu} \hat{F}^{v} d \sigma_{v}$ will appear in the right-hand side of Eqs. (9)-(12). This additive term will also appear in the expression of $v_{x}$ after Eq. (13) and in the denominator of Eq. (15) also.

The additional term, $\left(P_{\|}-P_{\perp}\right) \hat{F}^{\mu} \hat{F}^{v} d \sigma_{v}$, in Eq. (6) is orthogonal to $u^{\mu}$ (by definition of $\hat{F}^{\mu}$ ), so when we calculate the scalar product (16) their cross term vanishes, so

$$
\begin{aligned}
A_{0}^{\mu} A_{0 \mu}= & w_{\perp}\left(e-P_{\perp}\right)\left(u^{\mu} d \sigma_{\mu}\right)^{2}+\left(P_{\perp}\right)^{2}\left(d \sigma^{\mu} d \sigma_{\mu}\right) \\
& -\left(P_{\|}-P_{\perp}\right)\left(P_{\|}+P_{\perp}\right)\left(\hat{F}^{v} d \sigma_{v}\right)^{2}, \\
A_{0}^{\mu} d \sigma_{\mu}= & w_{\perp}\left(u^{\mu} d \sigma_{\mu}\right)^{2}-P_{\perp}\left(d \sigma^{\mu} d \sigma_{\mu}\right) \\
& +\left(P_{\|}-P_{\perp}\right)\left(\hat{F}^{v} d \sigma_{\nu}\right)^{2} .
\end{aligned}
$$

Now one can express $w_{\perp}\left(u^{\mu} d \sigma_{\mu}\right)^{2}$ from Eq. (24), and inserting it to Eq. (23) we obtain that

$$
\begin{aligned}
A_{0}^{\mu} A_{0 \mu}= & \left(e-P_{\perp}\right) A_{0}^{\mu} d \sigma_{\mu}+e P_{\perp}\left(d \sigma^{\mu} d \sigma_{\mu}\right) \\
& -\left(e+P_{\|}\right)\left(P_{\|}-P_{\perp}\right)\left(\hat{F}^{v} d \sigma_{\nu}\right)^{2},
\end{aligned}
$$

where this equation is not a scalar equation because it depends on $\hat{F}^{\mu}=C \Delta_{\mathrm{LR}}^{\mu \nu} d \sigma_{v}$, where the projector is dependent on $u^{\mu}$. These equations are similar to the ones obtained for the isotropic case; however, to solve this last equation we need a more complex relation among $e, P_{\|}$, and $P_{\perp}$. As these arise from the collision integral in the BTE approach, the needed relation may depend on $u_{0}^{\mu}$ and $d \sigma_{\mu}$. However, the escape probability may be simple, or may be approximated in a way that yields an ansatz for this relation with adjustable parameters, and then the problem is solvable. This was the case in Refs. [15,16].

The recent covariant formulation of the kinetic FO description [15] indicates that the relation among the different parameters of the anisotropic energy momentum tensor should be possible to express in terms of invariant scalars, which may facilitate the solution of the anisotropic FO problem.

When the adjustable parameters of the post-FO matter are determined in this way from the conservation laws, we still need the underlying anisotropic momentum distribution of the emitted particles to evaluate the final particle spectra using the Cooper-Frye formula with this anisotropic distribution function. Once again, when all conservation law equations are solved we have to check the nondecreasing entropy condition to see whether the selected FO hypersurface is realistic.

In case of an anisotropic final state, owing to the increased number of parameters and their more involved relations, 
the covariant treatment of the problem may not provide a simplification, compared to the direct solution of conservation laws for each component of the energy momentum tensor (e.g., [11,12]).

\section{Anisotropic initial and final states}

Recent viscous FD calculations evaluate the anisotropy of the momentum distribution is in the pre-FO viscous flow (see, e.g., [27].) This anisotropy is governed by the space-time direction of the viscous transport. The pre- and post-FO matter may still be different; for example, the pre-FO state may be viscous QGP with current quarks and perturbative vacuum, while post-FO we may have a hadron gas or constituent quark gas. The final state will also be anisotropic, not only because of the initial anisotropy but also owing to FO. The two physical processes leading to anisotropy are independent, so their dominant directions are in general different. In this case, the general symmetries are uncorrelated and cannot be exploited to simplify the description of the transition. Owing to the change of the matter properties, the conservation laws [Eqs. (1)-(3)] are needed to determine the parameters of the post-FO matter before the Cooper-Frye formula with nonequilibrium post-FO distribution is applied to evaluate observables.

\section{SUMMARY}

In this work a new simple covariant treatment is presented for solving the conservation laws across a transition hypersurface. This leads to a significant simplification of the calculation if both the initial and the final states are in thermal equilibrium. The same method can also be used for the more complicated anisotropic final state; however, this method is only advantageous if the more involved relations among the parameters of the post-FO distribution and the distribution itself is given in covariant form, preferably through invariant scalars.
[1] L. D. Landau, Izv. Akad. Nauk SSSR 17, 51 (1953).

[2] G. A. Milekhin, Zh. Eksp. Teor. Fiz. 35, 1185 (1958) [Sov. Phys. JETP 8, 829 (1959)]; Trudy FIAN 16, 51 (1961).

[3] F. Cooper and G. Frye, Phys. Rev. D 10, 186 (1974).

[4] G. F. Chapline, M. H. Johnson, E. Teller, and M. S. Weiss, Phys. Rev. D 8, 4302 (1973).

[5] W. Scheid, H. Müller, and W. Greiner, Phys. Rev. Lett. 32, 741 (1974).

[6] L. P. Csernai, Sov. JETP 65, 216 (1987) [Zh. Eksp. Teor. Fiz. 92, 379 (1987)].

[7] L. P. Csernai, Introduction to Relativistic Heavy Ion Collisions (Wiley, New York, 1994).

[8] A. H. Taub, Phys. Rev. 74, 328 (1948).

[9] V. K. Magas, L. P. Csernai, and D. D. Strottman, Phys. Rev. C 64, 014901 (2001); Nucl. Phys. A 712, 167 (2002).

[10] K. A. Bugaev, Nucl. Phys. A 606, 559 (1996).

[11] Cs. Anderlik, L. P. Csernai, F. Grassi, W. Greiner, Y. Hama, T. Kodama, Zs. I. Lazar, V. K. Magas, and H. Stöcker, Phys. Rev. C 59, 3309 (1999).

[12] K. Tamosiunas and L. P. Csernai, Eur. Phys. J. A 20, 269 (2004).

[13] Cs. Anderlik, Z. I. Lazar, V. K. Magas, L. P. Csernai, H. Stocker, and W. Greiner, Phys. Rev. C 59, 388 (1999); V. K. Magas, C. Anderlik, L. P. Csernai, F. Grassi, W. Greiner, Y. Hama, T. Kodama, Z. I. Lazar, and H. Stocker, Heavy Ion Phys. 9, 193 (1999); Phys. Lett. B 459, 33 (1999); Nucl. Phys. A 661, 596 (1999).

[14] V. K. Magas, A. Anderlik, C. Anderlik, and L. P. Csernai, Eur. Phys. J. C 30, 255 (2003).

[15] E. Molnar, L. P. Csernai, V. K. Magas, A. Nyiri, and K. Tamosiunas, Phys. Rev. C 74, 024907 (2006); J. Phys. G 34, 1901 (2007); E. Molnar, L. P. Csernai, and V. K. Magas, Acta Phys. Hung. A 27, 359 (2006); V. K. Magas, L. P. Csernai, and E. Molnar, ibid. 27, 351 (2006).
[16] V. K. Magas, L. P. Csernai, and E. Molnar, Eur. Phys. J. A 31, 854 (2007); Int. J. Mod. Phys. E 16, 1890 (2007); V. K. Magas and L. P. Csernai, Phys. Lett. B 663, 191 (2008); L. P. Csernai, V. K. Magas, E. Molnar, A. Nyiri, and K. Tamosiunas, Eur. Phys. J. A 25, 65 (2005); V. K. Magas, L. P. Csernai, E. Molnar, A. Nyiri, and K. Tamosiunas, Nucl. Phys. A 749, 202C (2005).

[17] F. Grassi, Y. Hama, S. S. Padula, and O. Socolowski, Phys. Rev. C 62, 044904 (2000).

[18] B. R. Schlei, Image Vision Comput. 27, 637 (2009).

[19] B. R. Schlei, Los Alamos National Laboratory Report No. LA-UR-03-3000, 37, 2003.

[20] B. R. Schlei, Los Alamos National Laboratory Report No. LA-UR-04-2143, 168, 2004.

[21] B. R. Schlei (unpublished).

[22] C. Perwass, Geometric Algebra with Applications in Engineering, Geometry and Computing (Springer, Berlin, 2009).

[23] L. Bravina, L. P. Csernai, P. Lévai, and D. Strottman, Phys. Rev. C 50, 2161 (1994).

[24] L. P. Csernai, Y. Cheng, Sz. Horvát, V. Magas, D. Strottman, and M. Zétényi, J. Phys. G 36, 064032 (2009).

[25] L. P. Csernai, Y. Cheng, V. K. Magas, I. N. Mishustin, and D. Strottman, Nucl. Phys. A 834, 261c (2010).

[26] S. B. Bass, A. Dumitru, M. Bleicher, A. Dumitru, M. Bleicher, L. Bravina, E. Zabrodin, H. Stocker, and W. Greiner, Phys. Rev. C 60, 021902 (1999); D. Teaney, J. Lauret, and E. V. Shuryak, Nucl. Phys. A 698, 479 (2002); S. A. Bass et al., J. Phys. G 34, S979 (2007); C. Nonaka, M. Asakawa, and S. A. Bass, ibid. 35, 104099 (2008); H. Petersen, J. Steinheimer, G. Burau, M. Bleicher, and H. Stöcker, Phys. Rev. C 78, 044901 (2008); T. Hirano and Y. Nara, ibid. 79, 064904 (2009).

[27] P. Huovinen and D. Molnar, Phys. Rev. C 79, 014906 (2009). 